\title{
New combinations and a complete list of Asparagus species in southern Africa (Asparagaceae)
}

\author{
A.C. FELLLINGHAM* and N.L. MEYER**
}

Keywords: Asparagaceac, Asparagus, new combinations, southem Africa, taxonomy

\author{
ABSTRACT
}

With only flower sexuality differing. Profasparagus can not be separated from Asparagus, neither at generic nor at subgeneric level. No significant differences in the degree to which filaments were connivent were observed between species placed under Myrsiphyllum and those under Prolasparagus/Asparagus. All southern African species of Asparagaceae are therefore reinstated under Asparagus and listed here without recognition of subgenera. Several new combinations are made.

\section{UITTREKSEL}

\begin{abstract}
Met slegs die geslagtelikheid van die hlomme wat verskil. kan Protasparagus nie van Asparagus geskei word nic, nóg op genus- nóg op subgenusvlak. Geen noemenswaardige verskille in die mate waartoe helmdrade saamneigend is, is waargeneem tussen spesies wat onder Myrsiphyllum geplaas is en die onder Profasparagus/Asparagus nie. Alle Suider-Afrikaanse spesies van Asparagaceae word dus heringestel onder Asparagus en hier gelys sonder erkenning van subgenusse. Verskeie nuwe kombinasies word gemaak.
\end{abstract}

The family name Asparagaceae was proposed by Jussieu (1789), soon after the genus Asparagus was named by Linnaeus (1753). The debate on the division of the genus into separate genera or subgenera. was started by Willdenow (1808) with his description of the genus Myrsiphyllum, which was countered by Roemer \& Schultes's (1829) treatment of Asparagus as a single genus. Kunth (1850) then not only subdivided the genus, but transferred it back to the Liliaceae. Baker (1875) continued the trend and it remained the standard treatment till Huber (1969) and Dahlgren \& Clifford (1982) once again advanced the concept of separate genera and the family name. Asparagaceae.

Obermeyer (1983. 1984) adopted Kunth's (1850) division of Asparagus s.l. into three separate genera: Myrsiphyllum Willd., Asparagus L. with unisexual flowers and Asparagopsis Kunth for the bisexual southern African species, in preference to Baker's (1875) single genus with three subgenera. Euasparagus Baker (= Asparagus). Asparagopsis (Kunth) Baker and Myrsiphyllum (Willd.) Baker. As Asparagopsis had been used by Montagne (1840) for a genus of the Rhodophycaceae and was therefore an illegitimate homonym in Kunth's application. Protasparagus Oberm. (1983) was published. This stance was also taken in the treatment of the family for the Flord of southerm Africa by Obermeyer \& Immelman (1992).

Malcomber \& Sebsebe (1993) came to the conclusion that. with only llower sexuality differing. Protasparagus and Asparagus could not be separated at generic level nor

*Stellenboxch Herbarium. National Botanical Institute, PO. Box 471. Stellentosch 7599.

**National Herharium. National Betanical Institute. Private Bag Xl(0). Preloria (x) (x)

MS. received: 1995-()2-21 even kept as separate subgenera. but rather, on the basis of the shared character of free filaments, Protasparagus and Asparagus belonged together in the subgenus Asparagus. Neither could Myrsiphyllum be a distinct genus on the strength of connivent filaments versus free ones in the rest of the Asparagaceae. This character was, however. sufficient to distinguish the taxon as a subgenus. They therefore proposed that Asparagus be the only genus in the Asparagaceae, with subgenera Myrsiphyllum and Asparagus, the latter including the species previously placed in Protasparagus.

We accept the treatment by Malcomber \& Sebsebe (1993) with reservation. The absence of $A$. juniperoides Engl. and A. multimberosus R.A. Dyer from the list of species investigated by these authors, prompted further investigation. As the close relatives of these two species are in the subgenus with connivent filaments. it could be expected to find the same condition here. However. it could not be demonstrated that filaments were markedly connivent, and no significant differences were observed in the degree to which filaments were connivent in the two subgenera. Therefore, we prefer to refrain from dividing the southern African species of the genus into subgenera.

In addition to the new combination made by Malcomber \& Sebsebe (1993). more new combinations as well as reinstatements are necessary for the southem African species of the genus Asparagus. The numbers next to the species names listed alphabetically below romb. nov. numbers indented), are the species numbers allocated by Obermever \& Immelman (1992): those in Mirsiphyllum being affixed with an ' $M$ '.

53. Asparagus acocksii Jessop in Bothalia 9: 74 (1966). Pronasparagus acencksii (Jerupp) Oberm.: 243 (1983): Oherm. \& Immelman: 57 (1992) 
46. Asparagus aethiopicus L., Mantissa plantarum: 32 (1767).

Protasparagus aethiopicus (L.) Oberm.: 243 (1983); Oberm. \& Immelman: 54 (1992).

19. Asparagus africanus Lam., Encyclopédie méthodique 1: 295 ( 1783 ).

Protasparagus africanus (Lam.) Oberm.: 243 (1983); Oherm. \& Immelman: 35 (1992).

48. Asparagus aggregatus (Oherm.) Fellingham \& N.L. Mev: comb. nov.

Promasparagus aggregatus Oherm. in Oherm. \& Immelman: 55 (1992).

M8. Asparagus alopecurus (Oberm.) Malcomber \& Sebsebe in Kew Bulletin 48: 73 (1993).

Myrsiphyllum alopecurum Oberm.: 85 (1984): Oberm.: 78 (1992).

50. Asparagus angusticladus (Jessop) Fellingham \& N.L. Mev. comb. nov.

Asparagus aethiopicus L. var. angusticladus Jessop: 69 (1966). Protasparagus angusticladus (Jessop) Oberm.: 243 (1983); (Oberm. \& Immelman: 56 (1992).

M2. Asparagus asparagoides $(L, W$. Wight. Century Dictionary 2: 845 (1909).

Medeola asparagoides L.: 339 (1753). Myrsiphyllum asparagoides (L.) Willd: 25 (1808); Oberm.: 78 (1984): Oberm.: 73 (1992).

51. Asparagus aspergillus Jessop in Bothalia 9: 71 (1966). Protasparagus aspergillus (Jessop) Oberm.: 243 (1983); Oberm. \& Immelman: 56 (1992).

2. Asparagus bayeri (Oberm.) Fellingham \& N.L. Mev: comb. nov.

Protasparagus baveri Oberm. in Oberm. \& Immelman: 21 (1992).

17. Asparagus bechuanicus Baker in W.T. ThiseltonDyer. Flora of tropical Africa 7: 429 (1898).

Protasparagus bechuanicus (Baker) Oterm. in Oberm. \& Immelman: 33 (1992)

63. Asparagus biflorus (Oberm.) Fellingham \& N.L. Mev: comb. nov.

Protasparagus biflorus (Oherm. in Oberm. \& Immelman: 63 (1992).

34. Asparagus buchananii Baker in Kew Bulletin 1893 $211(1893)$

Protasparagus buchananii (Baker) Oherm.: 243 11983); (Oherm. \& Immelman: 45 (1992).

8. Asparagus burchellii Baker in Joumal of the Linnean Society of London. Botany 14: 618 (1875).

Protasparagus burchellii (Baker) Oberm. in Oberm. \& Immelman $27(1992)$

4a. Asparagus capensis $I$. var. capensis. Species plantarum. edn 1: 314 (1753).

Protasparagus capensis (L.) Oherm.: 243 (1983): (herm. \& Immkl man: 23 (1992). 4b. Asparagus capensis L. var. litoralis Suess. \& J. Karl in Mitteilungen der Botanischen Staatssammlung, München 1: 50) (1950); Jessop: 44 (1966).

Protasparagus capensis L. var. litoralis (Suess. \& J. Karl) Oterm. comb. illeg. in Oherm. \& Immelman: 24 (1992).

47. Asparagus clareae (Oberm.) Fellingham \& N.L. Mev: comb. nov.

Prolasparagus clareae Oherm. in (Oberm. \& Immelman: 54 (1992).

45. Asparagus coddii (Oberm.) Fellingham \& N.L. Mey. comb. nov.

Protasparagus coddii Oherm. in (Oterm. \& Immelman: 53 (1992).

59. Asparagus concinnus (Baker) Kies in Bothalia 6: 178 (1951a).

Asparagus africunus Lam. var. concinmus Baker: 619 (1875). Protasparagus concinnus (Baker) Oberm. \& Immelman: 6l (1992).

41. Asparagus confertus $K$. Krause in Botanische Jahrbücher 51: 449 (1914).

Protasparagus confertus (K. Krause) Oberm. in Oherm. \& Immelman: $51(1992)$

18. Asparagus cooperi Baker in Gardeners' Chronicle 1: $818(1874 a)$.

Pronasparagus cooperi (Baker) Oberm.: 243 (1983): Oberm. \& Immeiman: 33 (1992).

52. Asparagus crassicladus Jessop in Bothalia 9: 75 (1966).

Protasparagus crassicladus (Jessop) Oherm.: 243 (198.3): Oherm. \& Immeiman: 57 (1992).

M9. Asparagus declinatus $L$., Species plantarum. edn I: 313 (1753).

Myrsiphyllum declinatum (L.) Oberm.: 86 (1984): Oberm.: 78 (1992).

39. Asparagus densiflorus (Kunth) Jessop in Bothalia 9: 65 (1966).

Asparagopsis densiffora Kunth: 9 (1850). Protasparagus densiflorus (Kunth) Oherm. in Oherm. \& Immelman: 49 (1992).

21. Asparagus denudatus (Kunth) Baker in Journal of the Linnean Society of London. Botany 14: 606 (1875).

Asparagopsis denudatus Kunth: $82(1850)$. Profosporagus denudatus

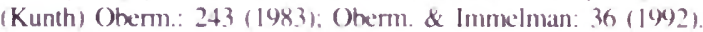

30. Asparagus devenishii (Oherm.) Fellingham \& N.L. Mey. comb. nov

Protasparagus devenishii Oberm. in Oterm. \& Immelman; 41 (19)2).

69. Asparagus divaricatus (Oberm.) Fellingham \& N.L. Mev: comb. nov.

Protasparagus divaricatus Ohern. in Oherrm. \& Immolmall $67(f(x) 2)$.

29. Asparagus edulis (Oberm.) Fellingham \& N.L. Mey. comb. nov.

Prifasparagus edulis Oberm. in Obenn. \& Immkelman: $41(\mid(x) 2)$.

10. Asparagus exsertus (Oberm.) Fellingham \& N.L. Mev: comb. nov.

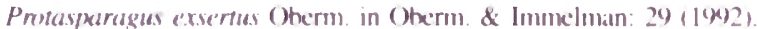


32. Asparagus exuvialis Burch., Travels in the interior of southern Africa 1: 432 (1822).

Prinasparagus exwialis (Burch.) Oberm.: 244 (1983): Oherm. \& Immelman: 42 (1992).

32a. Asparagus exuvialis Burch. forma ecklonii (Baker) Fellingham \& N.L. Mey: comb. nov.

Asparagus ecklonii Baker: 615 (1875). Protasparagus exuvialis (Burch.) Oherm. forma ecklomii (Baker) Oherm. in Oterm. \& Immelman: 43 (1992).

33. Asparagus falcatus $L$.. Species plantarum. edn $1: 313$ (1753).

Protasparagus falcatus (1.) Oherm.: 244 (1983): Oherm. \& Immelman: 43 (1992).

M 12. Asparagus fasciculatus Thunb.. Flora capensis. edn 2: $329(1823)$. (1992).

Myrsiphyllum fasciculatum (Thunh.) Oherm.: 87 (1984): Oherm.: 80

4(). Asparagus filicladus (Oberm.) Fellingham \& N.L. Mey. comb. nov.

Protasparagus filickedus Oberm. in Oberm. \& Immelman: 51 (1992).

1la. Asparagus flavicaulis (Oberm.) Fellingham \& N.L. Mev. comb. nov.

Protasparagus flavicaulis Oberm. in Oherm. \& Immelman: 29 (1992).

IIb. Asparagus flavicaulis (Oberm.) Fellingham \& N.L. Mey. subsp. setulosus (Oherm.) Fellingham \& N.L. Mev: comb. nov.

Protasparagus flavicaulis Oherm. subsp. sefulosus Oherm. in Oherm. \& Immelman: 30 (1992).

61. Asparagus fouriei (Oberm.) Fellingham \& N.L. Mev: comb. nov.

Protasparagus fouriei Oterm. in Oterm. \& Immelman: 62 (1992).

28. Asparagus fractiflexus (Ohenn.) Fellingham \& N.L. Mev: comb. nov.

Pronasporagus fractiflexus (term. in Oherm. \& Immelman: 40 (1992)

3. Asparagus glaucus Kies in Bothalia 6: 229 (1951b). Pronasparagus glancus (Kies) Oherm.: 224 (1983): Oherm. \& Immelman: 23 (1992)

31. Asparagus graniticus (Oberm.) Fellingham \& N.L. Mel: comb. nov.

Pnsasparagus granificus Oterm. in Oherm. \& Immelman: 42 (1992,

68. Asparagus intricatus (Oherm.) Fellingham \& N.L. Me'l comb. nov.

Profasparagus imricatus Oherm. in Oterm. \& Immciman: 65 (1992)

M7. Asparagus juniperoides Engl. in Botanische Jahrbücher 10: 3 (1889).

Mrsiphillum juniperoides (Engl.) Oherm.: 84 (1984): Otemm.: 78 (1092)

M4. Asparagus kraussianus (Kumb) J.F. Macbr in Contributions from the Gray Herbarium 56: 17 (1918).

Mirsiphyllum kraussianum Kunth: 107 (1850): Oherm: 80) (1984) (oterm: 76 (1992) .
42. Asparagus krebsianus (Kunth) Jessop in Bothalia 9: 74 (1966).

Asparagopsis krebsiana Kunth: 93 (1850). Protasparagus krebsianus (Kunth) Oberm.: 24 (1983): Oherm. \& Immelman: 51 (1992).

16. Asparagus laricinus Burch.. Travels in the interior of southern Africa 1: 537 (1822).

Pnnasparagus laricinus (Burch.) Oberm.: 244 (1983); Oberm. \& Immelman: 33 (1992).

23. Asparagus lignosus Burm.f.. Prodromus florae capensis: 10 (1768).

Pronasparagus lignosus (Burm.f.) Oberm. in Oberm. \& Immelman 37 (1992).

20. Asparagus longicladus N.E. Br. in Kew Bulletin 1921: 298 (1921).

Protasparagus longicladus (N.E. Br.) B. Mathew: 181 (1989): (Oterm. \& Immelman: 36 (1992)

36. Asparagus lynetteae (Oherm.) Fellingham \& N.L. Mev: comb. nov.

Pmiasparagus linetlecie Oherm. in Oherm. \& Immelman: 47 (1992).

60). Asparagus macowanii Baker in Journal of the Linnean Society of London. Botany 14: 609 (1875).

Prolasparagus macowanii (Baker) Oberm.: 244 (1983); Oherm. \& Immelman: 62 (1992).

6. Asparagus mariae (Oherm.) Fellingham \& N.L. Mev: comb. nov.

Protasparagus mariae Oherm. in Oterm. \& Immelman: 24 (1992).

57. Asparagus microraphis (Kunth) Baker in Journal of the Linnean Society of London. Botany 14: 612 (1875).

Asparagopsis microraphis Kunth: 83 (1850). Protasparagus mi croraphis (Kunth) Oherm.: 241 (1983): Oterm. \& Immelman: 59 (1992)

64. Asparagus minutiflorus (Kunth) Baker in Journal of the Linnean Society of London. Botany 14:616 (1875).

Asparagopsis minuifforus Kunth: 89 (1850). Protasparagus minuni florus i Kunth) (Oterm.: 244 (1983): Oherm. \& Immelman: 64 (1992).

15. Asparagus mollis (Oberm.) Fellingham \& N.L. Mev: comb.nov.

Protaspuragus mollis Oherm. in Oherm. \& Immelman: 32 (1992).

25. Asparagus mucronatus Jessop in Bothalia 9: $56(1966)$. Pronasparagus mucronahus (Jessop) Otherm. in Oterm. \& Immelman: $38(1042)$.

27. Asparagus multiflorus Baker in Joumal of the Linnean Society of London. Botany 14: 610 (1875).

Prolasparagus mulhiflones (Baker) Oterm): 24 (1983): Otherm. \& Immximan: $39(1992)$.

M1. Asparagus multituberosus R.A. Dyer in Bothalia 6: 42 (1954).

Mrsiphillum mulsituherosum (R.A. Dver) Oberns: 77 (1984): Oternil: 73,19621 
49. Asparagus natalensis (Baker) Fellingham \& N.L. Mev: comb. nov.

Asparagus aethiopicus L. var. natalensis Baker: 272 (1896). Prot asparagus natalensis (Baker) Oberm.: 244 (1983); Oherm. \& Immelman: 56 (1992).

37. Asparagus nelsii Schinz in Bulletin de l'Herbier Boissier, Sér. 1.4. App. 3: 44 (1896).

Protasparagus nelsii (Schinz) Oberm.: 244 (1983); Oberm. \& Immelman: $48(1992)$

56. Asparagus nodulosus (Oherm.) Fellingham \& N.L. Mev: comb. nov.

Protasparagus nodulosus Oberm. in Oberm. \& Immelman: 59 (1992).

12. Asparagus oliveri (Oberm.) Fellingham \& N.L Mev: comb. nov.

Protasparagus oliveri Oberm. in Oberm. \& Immelman: 30 (1992).

M3. Asparagus ovatus T.M. Salter in Journal of South African Botany 6: 167 (1940).

Myrsiphyllum ovatum (T.M. Salter) Oberm.: 79 (1984); Oberm.: 75 (1992).

38. Asparagus oxyacanthus Baker in Journal of the Lin nean Society of London. Botany 14: 625 (1875).

Protasparagus onacanthus (Baker) Oberm.: 244 (1983); Oberm. \& Immelman: 48 (1992).

22. Asparagus pearsonii Kies in Bothalia 6: 179 (1951a). Protasparagus pearsonii (Kies) Oberm.: 244 (1983): Oberm. \& Immelman: 37 (1992)

14. Asparagus pendulus (Oherm.) Fellingham \& N.L Mev: comb.nov.

Protasparagus pendulus Oberm. in Oberm. \& Immelman: 32 (1992)

55. Asparagus plumosus Baker in Journal of the Linnean Society of London. Botany 14: 613 (1875).

Protasparagus plumosus (Baker) Oberm.: 244 (1983); Oherm. \& Im melman: 59 (1992)

35. Asparagus racemosus Willd.. Species plantarum edn 4.2: 152 (1799).

Protasparagus racemosus (Willd.) Oberm.: 24 (1983); Oberm. \& Immelman: 45 (1992)

M11. Asparagus ramosissimus Baker in Gardeners Chronicle new series 2: 6(1874b). (1992).

Myrsiphyllum ramosissimum (Baker) Oterm: 87 (1984): ()herm: 79

1. Asparagus recurvispinus (Oberm.) Fellingham \& N.L. Mev: comb. nov.

Protcasparagus recuntispinus Oberm. in (Obern. \& Immelman: 19 (1992).

26. Asparagus retrofractus $L$. Species plantarum. edn I: 313 (1753).

Protasparagus retrofractus (L.) Oherm.: 2H 11983): (herm. \& Immelman: 39 (1992).

65. Asparagus rigidus Jessop in Bothalia 9: 64 (1966).

Protasparagus rigidus (Jesmp) Oterm.: 24 (1983): Oberm \& Immelman: 64 (1992)
24. Asparagus rubicundus P.J. Bergius, Descriptiones plantarum ex Capite Bonae Spei: 88 (1767).

Protasparagus rubicundus (P.J. Bergius) Oberm.: 244 (1983): Oberm. \& Immelman: 38 (1992).

M10. Asparagus scandens Thunb., Prodromus plantarum capensium 1: 66 (1794).

Mvrsiphvllum scundens (Thunh.) Oberm.: 86 (1984): Oberm: 79 (199?).

44. Asparagus schroederi Engl. in Botanische Jahrbücher 32: 97 (1903)

Protasparagus schroederi (Engl.) Oterm.: 244 (1983); Oherm. \& Immelman: $53(1992)$.

62. Asparagus sekukuniensis (Oberm.) Fellingham \& N.L. Mev: comb. nov.

Protasparagus sekukuniensis Oberm. in Oberm. \& Immelman: 63 (1992)

54. Asparagus setaceus (Kunth) Jessop in Bothalia 9: 51 (1966).

Asparagopsis setacea Kunth: 82 (1850). Protasparagus setaceus (Kunth) Oberm.: 244 (1983); Oherm. \& Immelman: 58 (1992).

9. Asparagus spinescens Steud. ex Roem. \& Schult., Systema vegetabilium 7.1: 334 (1829).

Prolasparagus spinescens (Steud. ex Roem. \& Schult.) Oberm. in Oberm. \& Immelman: 27 (| $(992)$

58. Asparagus stellatus Baker in Journal of the Linnean Society of London. Botany 14: 612 (1875).

Protasparagus stellatus (Baker) Oherm. in Oberm. \& Immelman; 61 $(1992)$.

5. Asparagus stipulaceus Lam., Encyclopédie méthodique 1: $297(1783)$.

Profasparagus stipulaceus (Iam.) Ohxerm.: 244 (198.3); Oberm. \& Immelman: 24 (1992).

66. Asparagus striatus (L.f.) Thunb., Prodromus plantarum capensium 1: 65(1794)

Dracaena siriata L.f: 204 (1781). Protasparagus striatus (L.f.) Oherm: 24 (1983): Oherm \& Immkalman: 64 (1992).

7. Asparagus suaveolens Burch.. Travels in the interior of southern Africa 2: 226 (1824).

Protasparagus suweolens (Burch.) Oberm.: 244 (1983); (Oberm. \& Immelman: 25 (1992)

67. Asparagus subulatus Thumb. Prodromus plantarum capensium 1: 66 (1794).

Protaspuragus subukatus (Thunh.) Oterm.: 244 (1983); Oberm. \& Immelman: $65(199)$ ?)

43. Asparagus transvaalensis (Oberm.) Fellingham \& N.L. Mev: comb. nov.

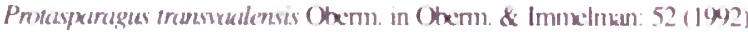

M6. Asparagus undulatus (L.f.) Thunb., Prodromus plantarum capensium 1: 66 (1794)

Dracaena undulata L.f.: 203 (1781). Mirsiphyllum undularum (I.i.) kiunth: $1(99,(18.50)$ ): Oherm: 83 (1984); Oherm: 77 (1992). 
13. Asparagus virgatus Baker in Refugium botanicum 3: t. $214(1870)$ (1992).

Protasparagus virgatus (Baker) Oherm. in Oherm. \& Immelman: 31

M5. Asparagus volubilis Thunh., Prodromus plantarum capensium 1: 66 (1794).

Mvrsiphyllum volubile (Thunh.) Oberm.: 82 (1984): Oberm.: 76 (1902).

\section{ACKNOWLEDGEMENTS}

We gratefully acknowledge the help and guidance received from Mr E.G.H. Oliver in the preparation of this article.

\section{REFERENCES}

BAKER. J.G. 1870. Asparagus virgatus. In W.W. Saunders, Refugium bolanicum 3: t. 214. Van Voorst. London.

BAKER, J.G. 1874a. New garden plants: Asparagus (Aspuragopsis) cooperi Baker. Gardeners' Chronicle 1: 818.

BAKER. J.G. 1874h. Gardeners' Chronicle new series 2: 6.

BAKER. J.G. 1875. Revision of the genera and species of Asparagaceac Journal of the Linnean Society of London. Botany 14: 59.4-631.

BAKER. J.G. 1893. Decades kewenses-Decas VI. Kew Bulletin 1893 209-212.

BAKER. J.G. 1896. Asparagus. In W.T. Thiselton-Dyer, Flora capensis 6: 256-274. Reeve, London.

BAKER. J.G. 1898. Asparagus. In W.T. Thiselton-Dyer. Flora of iropical Africa 7: 425-436. Reeve, London.

BERGIUS, P.J. 1767. Descriptiones plantarum ex Capise Bonat Spei Salvius, Stoxkholm

BROWN. N.E. 1921. New plants from tropical and South Africa collected by Archdeacon F.A. Rogers. Kew Bulletin 1921: 289-301.

BURCHELL. W.J. 1822. Travels in the interior of southem Africa 1. Longman, London.

BURCHELI. W.J. 1824. Travels in the interior of southern Africa 2 Longman, London.

BLRMAN. N.1. 1768. Prodromus florae copensis in Flora indica. Haak. leiden.

DAHL.GREN, R.M.T. \& CLIFFORD. H.T. 1982. The Monocotyledons: a comparative study. Academic Press. London.

DYER, R.A. 1954. Liliaceac. Bothalia 6: 44) $\$ 45$

ENGLER. A. 1889. Plantac Marlothianae. Liliaceac. Bontamische Johrbiucher 10:2, 3

ENGLER. H.G.A. 19013. I.liaceate africanac II. Boranische Jahrbücher 32: 89-97.

HUBER. H. 1969. Dic Samenmerkmale und Verwandtschaftsverhältnisse der Lilibloren. Minteilungen der Bonanischen Stablssummlung. Miunchen 8: 219-538
JESSOP, J. 1966. The genus Asparagus in southern Africa. Bothalia 9: $31-96$.

JUSSIEU. A.L. DE 1789. Genera plantarum. Paris.

KIES, P. 1951a. Notes on Asparagus africanus and related species. Bothalia 6: 177-181.

KIES. P. 1951b. Newly described species. Bothalia 6: 213-238.

KRAUSE. K. 1914. Liliaceae africanae V. Botanische Jahrbücher 51: 440-450.

KUNTH. C.S. 1850. Enumeratio plantarum 5. Cotta. Stuttgart \& Tübingen.

LAMARCK. J.B.A.P.M. DE 1783. Encuclopédie mérhodique 1. Mme veuve Agasse. Paris.

LINNAEUS. C. 1753. Species plantarum. edn 1. Salvii. Stockholm.

LINNAEUS. C. 1767. Mantissa plantarum. Salvii. Stockholm.

LINNAEUS. C. VON (fil.) 1781. Supplementum plantarum. Impensis Orphanotrophei. Brunswick.

MACBRIDE. J.F. 1918. Further new or otherwise interesting Liliaceac. Consributions from the Gray Herbarium of Hanard University 56: 17.

MALCOMBER. S.T \& SEBSEBE. D. 1993. The status of Protasparagus and Myrsiphyllum in the Asparagaceae. Kew Bulletin 48: 63-78.

MATHEW, B. 1989. Notes on tropical African Asparagaceae. Kew Bulletin H: 181, 182 .

MONTAGNE. J.P.F.C. 1840). Aspuragopsis. In P.B. Webb \& S. Berthelot, Histoire Naturelle des lles Canaries 3.2: 15. Béthune, Paris.

OBERMEYER, A.A. 1983. Protasparagus Oberm. nom. nov.: new combinations. South African Joumal of Botany 2: 243, 244.

OBERMEYER. A.A. 1984. Revision of the genus Myrsiphyllum Willd. Bothalia 15: 77-88

OBERMEYER. A.A 1992. Mvrsiphwllum. In O.A. Leistner. Flora of southem Africa 5.3: 71-82.

OBERMEYER. A.A. \& IMMELMAN, K.L. 1992. Protasparagus. In O.A. Leistner, Flora of southerm Africa 5.3: 11-70.

ROEMER. J.J. \& SCHULTES. J.A. 1829. Camli a Linné equitis Systema vegerabilium 7.1. Cotta. Stuttgan.

SALTER. T.M. 1940. Plantac novac Africanae XV. Joumal of South African Botany 6: $165-175$

SCHINZ. H. 1896. Die Pflanzenwelt Deutsch-Südwestafrikas. Bullerin de l'Herbier Boissier Sér. 1.4. App. 3: 1-57.

SUESSENGUTH. K. \& KARL. J. 1950. Diagnoses novae plantarum in Africa australi a Dr S. Rehm et aliis collectarum. Misteilungen der Botanischen Stautssammlung. München 1: 50.

THUNBERG. C.P. 1794. Prodromus plantarum capensium 1. Edman. U'ppsala.

THUNBERG. C.P. 1823. Flora capensis, ed. Schultes. Edman. Uppsala.

WIGHT. W.F. 1909 . Cenur Dictionan 2: 845.

WIL.DENOW. C.L. 1799. Caroli a Linné Species plantarum editio quaria 2. Berlin.

WILLDENOW. C.L. 1808 , Nähere Bestimmung einiger Liliengewächse. besonders aber derienigen. die zur sechsten Klasse und dritten Ordnung des I innéxchen Systems gehoren. Gesellschafi naturforschender Freunde zu Berlin Maga-in 2: 25, 26. 